\title{
Proton Decay from Excited States in Spherical Nuclei *
}

\author{
Ş. Mişicu, \\ National Institute for Nuclear Physics and Engineering, Bucharest-Măgurele, POB MG-6, \\ Romania, \\ N. Cârjan and P. Talou \\ Centre d'Etudes Nucléaires de Bordeaux Gradignan, BP 120 - F 33175 Gradignan Cedex, \\ France
}

(May 1998)

\begin{abstract}
Based on a single particle model which describes the time evolution of the wave function during tunneling across a one dimensional potential barrier we study the proton decay of ${ }^{208} \mathrm{~Pb}$ from excited states with non-vanishing angular momentum $\ell$. Several quantities of interest in this process like the decay rate $\lambda$, the period of oscillation $T_{o s c}$, the transient time $t_{t r}$, the tunneling time $t_{t u n}$ and the average value of the proton packet position $r_{a v}$ are computed and compared with the WKB results.
\end{abstract}

23.50.+z, 21.60.Gx

Typeset using REVTEX

*Work partially supported by the IN2P3-IFIN-HH Collaboration grant no.95-17 


\section{INTRODUCTION}

The recent interest in nuclei beyond the proton drip line, prompted the study of proton emission phenomenon from the ground state of such nuclei [1]. Like in the case of $\alpha$ decay, this process consists in the tunneling of the proton across a potential barrier. However for this kind of radioactivity it is vital to add the centrifugal potential to the Coulomb barrier since the majority of emitted protons are likely to decay from states with $\ell \neq 0$.

In last years a series of theoretical investigations, based on the quasi-classical approximation have been carried out in this field [2 4 ]. The proton half-lives of observed heavy proton emitters were calculated and compared with the experimental ones and in some cases a good agreement was found.

In the Gamow approach the decay is treated as a stationary process, the penetrability being given by the ratio of probabilities of finding the quantum particle on each side of the potential barrier. In this image the dynamical aspects are neglected. However quantities like the tunneling time are important in decay processes, in fission or in fusion reactions. In order to include the time evolution of a wave packet propagating in a classically forbidden region one need to solve the time dependent Schrödinger equation (TDSE). There have been some attempts to incorporate the time-dependency in the study of the proton decay [5] by expanding the wave function into a term describing the bound-state of the prepared nucleus $\psi_{0}(A)$ and a set of exit channel wave functions $\chi$ orthogonal to it which decomposed into the intrinsic states of the daughter nucleus $\psi(A-1)$ and of the proton $\phi_{p}$. However, imposing that the decay width is smaller than the resonance (quasi-stationary) energy $\left(\Gamma \ll E_{0}\right)$ the treatment reduce to the stationary Schrödinger equation for $\psi_{p}(r, t=0)$ with a complex energy $E_{0}-i \Gamma / 2$. Afterwards the usual computational procedure is carried on [6].

In this paper, based on a previous application of TDSE to the study of $\alpha$ decay [7,8], we address the question of proton-decay from orbitals with $\ell \neq 0$ for the spherical nucleus ${ }^{208} \mathrm{~Pb}$. This process is different from the ground-state proton emission beyond the proton drip line. However the theoretical techniques that we employ in this paper can be applied 
in both cases without major changes. In our time-dependent approach the description of the decaying system is fully contained in the state vector. The time evolution of this vector enables us to determine the decay probability at any moment $t$. We are interested to study the influence of the potential on the tunneling quantities and to determine to what extent the errors of the WKB method depends on the energy of the quasi-stationary state and on the angular momentum.

\section{DYNAMICAL APPROACH TO QUANTUM TUNNELING}

The interaction between the proton and the daughter nucleus ${ }^{207} \mathrm{Tl}$ is described by an average Woods-Saxon (WS) field which accounts for the nuclear potential

$$
V_{N}(r)=-\frac{V_{0}}{1+\exp \left(\frac{r-R_{0}^{N}}{a}\right)}
$$

a Coulomb potential, which is approximated by the interaction between the point proton and the uniformly charged spherical core of charge $Z-1$

$$
\begin{array}{rlrl}
V_{C}(r) & =-\frac{(Z-1) e^{2}}{R_{0}^{C}}\left[1+\frac{1}{2}\left(1-\left(\frac{r}{R_{0}^{C}}\right)^{2}\right)\right], r \leq R_{0}^{C} \\
& =\frac{(Z-1) e^{2}}{r}, & r \geq R_{0}^{C}
\end{array}
$$

and the centrifugal barrier

$$
V_{c f}(r)=\frac{\hbar^{2}}{2 \mu r^{2}} \ell(\ell+1)
$$

The WS interaction is defined by the nuclear radius $R_{0}{ }^{N}=r_{0} A^{1 / 3}$, with $r_{0}=1.25 \mathrm{fm}$, the diffuseness $a=0.7 \mathrm{fm}$, and the depth of the central potential $V_{0}=58 \mathrm{MeV}$ [9]. Note that in other papers, the depth of the potential is not fixed, its value being adjusted to reproduce the experimental energy of the quasi-bound state [3, 4]. The Coulomb radius is given by $R_{0}^{C}=r_{0}(A-1)^{1 / 3}$.

The initial wave function of the proton was chosen to correspond to a quasi-stationary state of the potential $V(r)=V_{N}(r)+V_{C}(r)+V_{c f}(r)$, with positive energy $E>0$. The decay 
width of such a metastable state can be calculated using an analytic method developed by Gurwitz et al. [10]. In this approach it is assumed that the proton occupies a bound eigenstate in the potential well $V(r)+\varepsilon(r)$ which represents a slight modification of $V(r)$. Therefore we take

$$
\psi_{p}(r, t=0)=\phi_{n l}^{(V+\varepsilon)}
$$

where, $\phi_{n}^{(V+\varepsilon)}$ is an eigenstate of energy $E_{n \ell}^{(V+\varepsilon)}$ corresponding to the Hamiltonian

$$
H_{0}=-\frac{\hbar^{2}}{2 \mu} \Delta+V(r)+\varepsilon(r)
$$

where $\mu$ is the reduced mass of the daughter-proton system. The modification $\varepsilon(r)$ reads

$$
\begin{aligned}
\varepsilon(r) & =V\left(r_{\max }\right)+\left(r-r_{\max }\right) \tan \theta-V(r), & & r \geq r_{\max } \\
& =0, & & r \leq r_{\max }
\end{aligned}
$$

where $\theta$ gives the slope of the potential barrier beyond the point $r_{\max }$ at which $V(r)$ attain its maximum.

In what follows we shall consider only the wave functions $\phi_{n \ell}^{(V+\varepsilon)}$ with the highest eigenvalue $E_{n \ell}^{(V+\varepsilon)}$ bellow the barrier $V_{B}=V\left(r_{\max }\right)$.

In Table I we list the heights of the barriers $\left(V_{B}\right)$, their locations $\left(r_{\max }\right)$ and the selected eigenvalues $\left(E_{n \ell}\right)$ for a given value of the angular momentum $\ell$.

The next step consists in the resolution of the time dependent Schrödinger equation:

$$
i \hbar \frac{\partial}{\partial t} \psi_{p}(r, t)=H(r) \psi_{p}(r, t)
$$

where

$$
H(r)=-\frac{\hbar^{2}}{2 \mu} \Delta+V(r)
$$

A numerical procedure based on the iterated leap-frog method, provides the solution of this equation [11]. The equidistant spatial grid used in this method is large enough such that no interference of the outgoing and ingoing wave functions will take place during the time 
intervals employed in the calculations. Once we get the wave-function which describes the time evolution of the proton packet through the potential barrier we are able to compute relevant quantities of the decay process.

The tunneling probability can be expressed as the probability of finding the proton beyond a certain point $r_{B}$ on the border which separates the zone inside the barrier from the external one

$$
P_{T D}\left(r_{B}, t\right)=\int_{r_{B}}^{\infty}\left|\psi_{p}(r, t)\right|^{2} r^{2} d r
$$

The decay rate is calculated according to the relation

$$
\lambda_{T D}\left(r_{B}, t\right)=\frac{\dot{P}_{T D}}{1-P_{T D}}
$$

It is also interesting to calculate the average value of the proton wave packet position operator, $r_{a v}$, inside the nucleus

$$
r_{a v}\left(r_{B}, t\right)=\frac{\int_{0}^{r_{B}} r\left|\psi_{p}(r, t)\right|^{2} r^{2} d r}{\int_{0}^{r_{B}}\left|\psi_{p}(r, t)\right|^{2} r^{2} d r}
$$

\section{NUMERICAL RESULTS AND COMPARISON WITH WKB}

In Figure $1 \mathrm{a}, \mathrm{b}$ we present the time evolution of the proton wave function $\psi_{p}(r, t)$ for two angular momenta $l=0,5$ at four different moments. We see the tendency of the wave function to decrease its amplitude in the interior of the barrier when time goes on. Eventually we observe that the fraction of the wave function which passed across the barrier behaves like a spreaded wave packet (at least on the spatial interval that we considered, i.e. up to $60 \mathrm{fm}$ ). This tendency is more pronounced for the wave function with $\ell=5$, because it faces a thiner barrier (see Table 2). It is worthwhile to mention that although the wave function amplitude decreases constantly in time its shape does not change to much inside the barrier.

In figures $2 \mathrm{a}, \mathrm{b}$ the time-dependent decay rate $\lambda_{T D}$ is ploted for angular momentum $\ell=0,2,5$ and 8 . As has been pointed in an earlier work [0] the decay rate undergoes 
two regimes. In the first one, $\lambda_{T D}$ oscillates but increases on the average. The fact that at small times the decay rate is not constant as characteristic for exponential decay, but varies with time is typical for a quantum mechanical description [12]. This fact contrasts to the usual classical image which portrays the radioactive system as an ensamble of nuclei decaying independently one of each other with a probability which does not depend on time. In the second regime $\lambda_{T D}$ performs small fluctuations around a constant value, that we call asymptotic value $\lambda_{T D}(\infty)$.

The decay rates presented in figure 2 a correspond to $r_{B}=11.6 \mathrm{fm}$, i.e. the inferior limit of integration is choosed to lay between the two turning points as can be observed from Table 2. If $r_{B}$ is increased, the irregularities occuring in the first regime are smoothed out. This fact is pictured in Figure $2 \mathrm{~b}$, where $r_{B}$ is choosed to be $25 \mathrm{fm}$.

In Figure 3 we represented the behaviour of the wave packet's average position inside a potential region defined by $r_{B}=25 \mathrm{fm}$. As in the case of the decay rate we deal with two regimes. Whereas in the first regime $r_{a v}$ increases up to a certain limit, in the second one it performs small-amplitude fluctuations around this limiting value as we expected since, according to a previous remark, the wave function does not change to much its shape. The period of oscillations in this second regime is denoted by $T_{\text {osc }}$ and its value can be deduced simply by measuring the distance between two maxima of $r_{a v}$ (right column in Fig.3). As has been noted in previous papers [7,8], the quasi-stationary state tends to penetrate the potential barrier by performing these small-amplitude oscillations instead of simply crossing the barrier from one side to the other. For this reason it makes sense to associate the frequency of collisions in the formula of Gamow with $1 / T_{\text {osc }}$ instead of $1 / T_{\text {cross }}$, which is defined by the quasi-classical expression (see eq.(13) bellow).

In the WKB approximation the decay rate $\lambda_{S T}$ is constant, its value resulting from the product of the barrier penetrability $p$ and the collision frequency $\nu$

$$
\lambda_{S T}=\nu \cdot p
$$

where $\nu$ is given by the inverse of the classical period of motion 


$$
T_{\text {cross }}=\frac{2 \mu}{\hbar} \int_{r_{t 1}}^{r_{t 2}} \frac{d r}{k(r)}
$$

the wave number $k(r)$ reading

$$
k(r)=\left[\frac{2 \mu}{\hbar^{2}}(Q-V(r))\right]^{1 / 2}
$$

and the penetrability

$$
p=\exp \left(-2 \int_{r_{t 2}}^{r_{t 3}} d r \sqrt{\frac{2 \mu}{\hbar^{2}}(V(r)-Q)}\right)
$$

The stationary states computed in the modified potential, become quasi-stationary when we turn on the real potential (without the modification $\varepsilon(r)$ ) and their energy is no longer well defined. Therefore, in all the above formulas the decay energy $Q$ was taken to be the energy of the quasi-stationary state $E_{0}$, computed as the average energy of the time dependent Scrödinger equation

$$
E_{0}=\left\langle\psi_{p}(r, t)|H(r)| \psi_{p}(r, t)\right\rangle
$$

In what concerns the decay rate, it can be inferred from Table 2 that the decay rates calculated in the time dependent approach $\lambda_{T D}$ are in relatively good agreement with the WKB values $\lambda_{S T}(\infty)$ especially when the quasi-stationary energy decreases with respect to the barrier height. Except the last case, with the highest angular momentum, in all other cases the WKB results overestimates the time-dependent values.

The comparison between the times $T_{\text {osc }}$ and $T_{\text {cross }}$ (see Table 2) shows that they have very close values. However they are refering to different types of motion, the first describing the small oscillations of the proton wave function between the walls of the barrier during tunneling, the second, the classical movement inside the potential well.

The transient time is defined as the time interval between the moment when $\lambda_{T D}$ starts to increase up to the moment when it reaches the limiting value $\lambda_{T D}(\infty)$. It depends on the energy of the quasi-stationary state. Its value can be deduced by inspecting figures 2 $\mathrm{a}$ and $\mathrm{b}$. The tunneling time is related to the shift in time of the transition point between 
the two above mentioned regimes, for the decay rate. Therefore it can be associated to the time necessary for the wave function to cross the barrier. Computing the decay rate for two different values of $r_{B}$, i.e. the barrier's turning points, and measuring the time delay between the two maxima of the two curves one gets the tunneling time (see Fig.4 a and b ). Notice that for $\ell=0,2,5$ the tunneling time decreases with increasing $\lambda_{T D}(\infty)$. However this does not happens for the state with higher angular momentum $\ell=8$, where, although the decay rate is smaller, the tunneling seems to take place faster. In fact the tunneling time is correlated with the imaginary time, which is nothing else than the time necessary for the proton packet to cross the inverse potential, i.e.

$$
t_{\text {imag }}=\int_{r_{t 2}}^{r_{t 3}} d r \sqrt{\frac{\mu}{2(V(r)-Q)}}
$$

\section{SUMMARY}

Motivated by recent theoretical and experimental investigations on proton radioactivity phenomenon, we studied the time dependent characteristics of the proton tunneling from excited states in the spherical nucleus ${ }^{208} \mathrm{~Pb}$. Although this process is different from the ground-state proton emission beyond the proton drip line, the approach employed in this paper could be easily extended to the above mentioned case of proton radioactivity.

Since other theoretical approaches are based on the semiclassical approximation we were interested to compare our exact results with the WKB ones. We found that the discrepancy between the two methods decreases when the difference between the top of the barrier and the energy of the quasi-stationary state increases. Our study does not concern a certain

angular momentum state which could be measured in the decay reaction ${ }^{208} \mathrm{~Pb}^{*} \rightarrow{ }^{207} \mathrm{Tl}+p$. Rather for a fixed set of Woods-Saxon parameters we investigated the dependence of the proton tunneling on time choosing one quasi-stationary state for every angular momentum. It seems that the accuracy of the WKB approximation increases with $\ell$. For a comparison with the experiment one should fit some of the WS parameters, e.g. the potential depth, in 
such a way to reproduce the observed energy. However the present comparison between the WKB and the time-dependent approaches gives an idea of the error involved in the stationary approach and provides a good starting point for future investigations of proton decay using TDSE which could eventually answer to some questions related to this phenomenon. 


\section{REFERENCES}

[1] Hofmann S 1989 Particle Emission from Nuclei, edited by M.Ivaşcu and D.N.Poenaru (CRC, Boca Raton, FL).

[2] Kadmenskii S G and Khlebostroev V G 1974 Sov.J.Nucl.Phys. 18505.

Bugrov V P and Kadmenskii S G 1989 Sov.J.Nucl.Phys. 49967.

[3] Buck B, Merchant A C and Perez S M 1992 Phys.Rev. C 451688.

[4] Aberg S, Semmens P B and Nazarewicz W 1997 Phys.Rev. C 561762.

[5] Feix W F and E R Hilf 1983 Phys.Lett. 120B 14.

[6] De Vries R M 1976 Comput.Phys.Commun. 11249.

[7] Serot O and Carjan N 1994 Zeit.Phys.A 349353.

[8] Serot O, Carjan N and Strottman H 1994 Nucl.Phys. A569 562.

[9] Rost E 1968 Phys.Lett.B 26184.

[10] Gurwitz S A and Kalbermann G 1987 Phys.Rev.Lett. 59262.

[11] Serot O 1992 Dissertation Thesis, CENBG:9216 (Université de Bordeaux I).

[12] Grotz K and Klapdor H V 1984 Phys.Rev. C 302098. 


\section{TABLES}

TABLE I. The values of the barrier heights $V_{B}$, their locations $r_{\max }$, the selected eigenvalues $E_{n \ell}$ and the wave functions number of nodes for different angular momenta $\ell$.

\begin{tabular}{ccccc}
\hline \hline Angular Momentum & Number of nodes & $r_{\max }(\mathrm{fm})$ & $V_{B}(\mathrm{MeV})$ & $E_{n \ell}(\mathrm{MeV})$ \\
\hline$\ell=0$ & 4 & 10.62 & 10.256 & 7.78 \\
$\ell=1$ & 3 & 10.55 & 10.631 & -0.04 \\
$\ell=2$ & 3 & 10.45 & 11.392 & 7.23 \\
$\ell=3$ & 2 & 10.32 & 12.560 & -2.06 \\
$\ell=4$ & 2 & 10.15 & 14.163 & 5.29 \\
$\ell=5$ & 2 & 10.00 & 16.233 & 12.53 \\
$\ell=6$ & 1 & 9.82 & 18.800 & 0.64 \\
$\ell=7$ & 1 & 9.62 & 21.916 & 8.44 \\
$\ell=8$ & 1 & 9.45 & 25.607 & 16.58 \\
\hline \hline
\end{tabular}

TABLE II. The quasi-stationary energies $E_{0}$, the turning points of the potential $\left(r_{t 1}, r_{t 2}, r_{t 3}\right)$, the asymptotic value of the decay rate $\lambda_{T D}(\infty)$ and its WKB correspondent $\lambda_{S T}$, the oscillation period $T_{o s c}$ and the crossing time $T_{\text {cross }}$.

\begin{tabular}{cccccccc}
\hline \hline$E_{0}(\mathrm{MeV})$ & $r_{t 1}(\mathrm{fm})$ & $r_{t 2}(\mathrm{fm})$ & $r_{t 3}(\mathrm{fm})$ & $\lambda_{T D}(\infty)\left(\mathrm{s}^{-1}\right)$ & $\lambda_{S T}\left(\mathrm{~s}^{-1}\right)$ & $T_{\text {osc }}(\mathrm{s})$ & $T_{\text {cross }}(\mathrm{s})$ \\
\hline 7.71 & - & 9.24 & 14.98 & $1.25 \times 10^{+20}$ & $1.74 \times 10^{+20}$ & $2.75 \times 10^{-22}$ & $2.95 \times 10^{-22}$ \\
7.19 & 1.70 & 8.90 & 17.15 & $1.90 \times 10^{+19}$ & $2.33 \times 10^{+19}$ & $2.60 \times 10^{-22}$ & $2.74 \times 10^{-22}$ \\
12.45 & 3.56 & 8.74 & 13.12 & $2.23 \times 10^{+20}$ & $2.59 \times 10^{+20}$ & $2.24 \times 10^{-22}$ & $2.43 \times 10^{-22}$ \\
16.55 & 5.31 & 7.89 & 13.69 & $2.79 \times 10^{+19}$ & $2.52 \times 10^{+19}$ & $1.50 \times 10^{-22}$ & $1.79 \times 10^{-22}$ \\
\hline \hline
\end{tabular}




\section{Figure Captions}

Fig. 1. Time evolution of the squared wave function $\left|\psi_{p}(r, t)\right|^{2}$ for angular momentum (a) $\ell=0$ and (b) $\ell=5$ at moments $t=0,1.5 \cdot 10^{-21}, 3 \cdot 10^{-21}$ and $4.5 \cdot 10^{-21}$ seconds. In the second case the barrier through which the proton undergoes tunneling is thiner and therefore the probability to find it outside the barrier at a later time is larger. The wave function amplitude decreases in time and later on, the part of the wave function which already tunneled manifests itself as a well-spreaded wave packet.

Fig. 2. The time dependent decay rates $\lambda_{T D}$ for the four selected quasi-stationary states of angular momentum $\ell=0,2,5,8$. In the eq.(10) we choosed (a) $r_{B}=11.6 \mathrm{fm}$ and (b) $r_{B}=25 \mathrm{fm}$. In all four cases we observe that after a certain time the decay rate will fluctuate around an asymptotic value. In the second case these fluctuations are smoother.

Fig. 3. The average value of the wave packet position operator $r_{a v}$ for three quasistationary states $(\ell=0,2,5)$. On the left side the asymptotic behaviour of $r_{a v}$ is observed. whereas on the right side we foccused on the small amplitude oscillations of the wave packet on its way to tunneling.

Fig. 4. The decay rates for (a) $\ell=0,2$ and (b) $\ell=5,8$ when $r_{B}$ is choosed to be the internal turning point (full line) and the external turning point (dotted line). The difference between the maxima of the two curves gives the tunneling time $t_{t u n}$. 

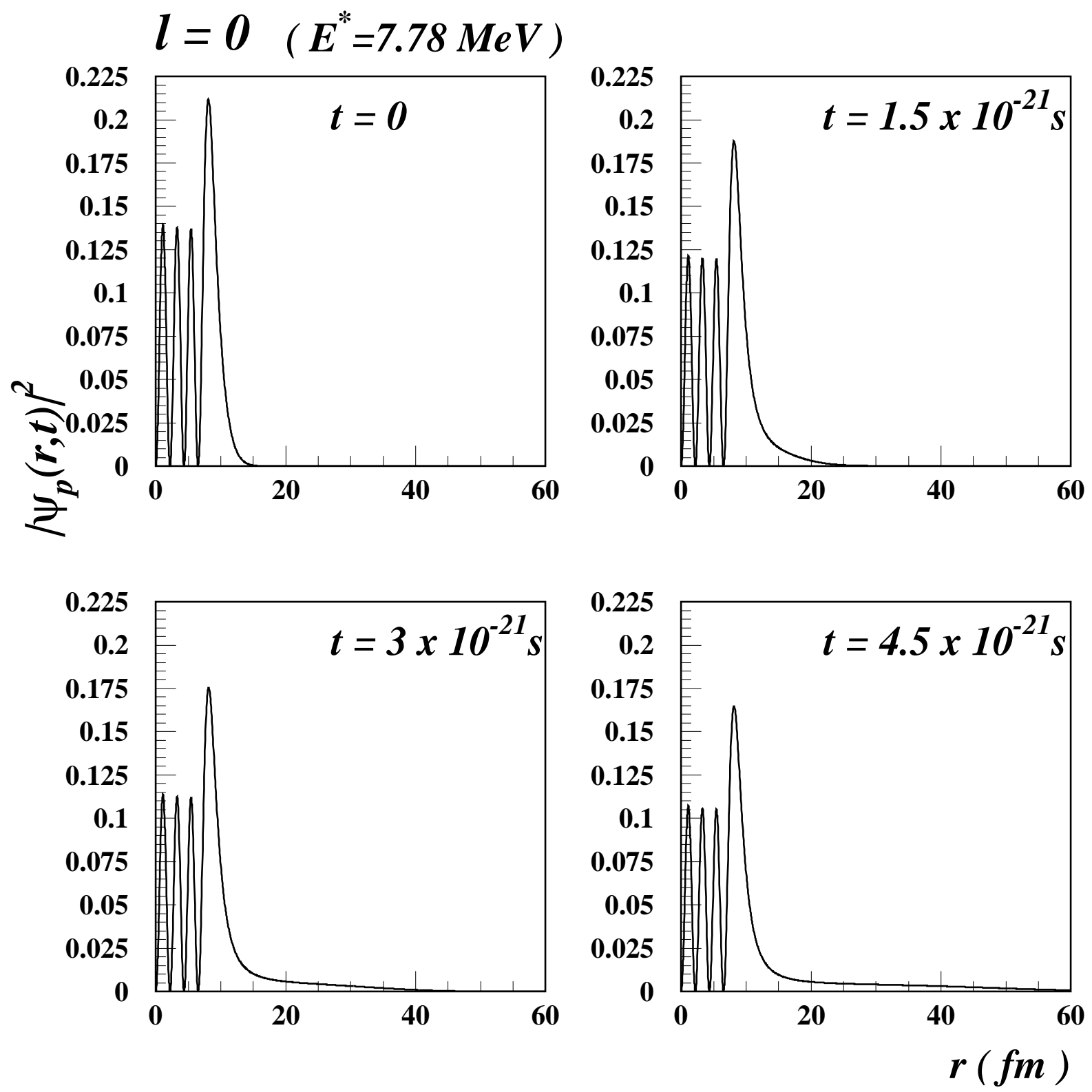


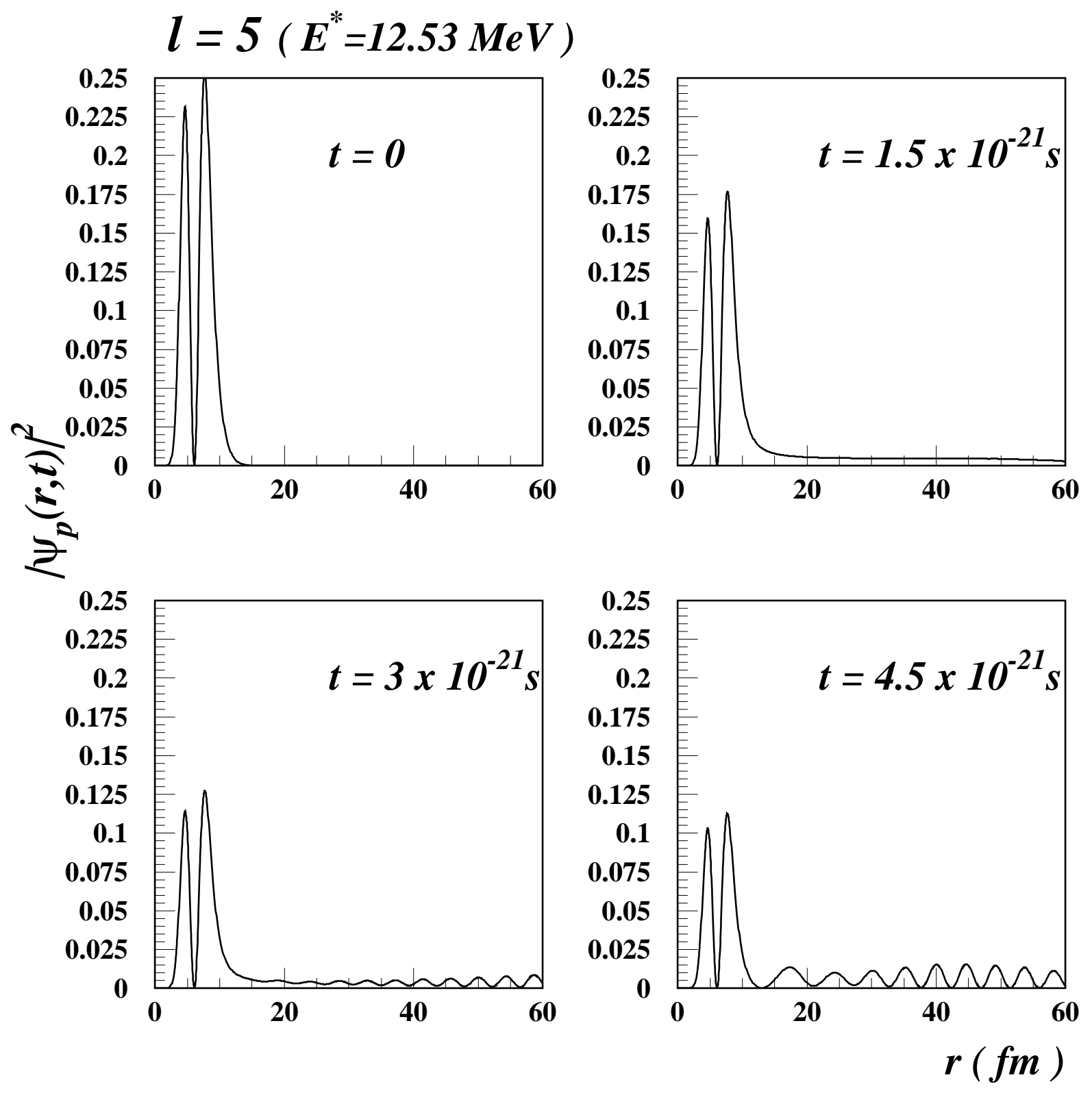



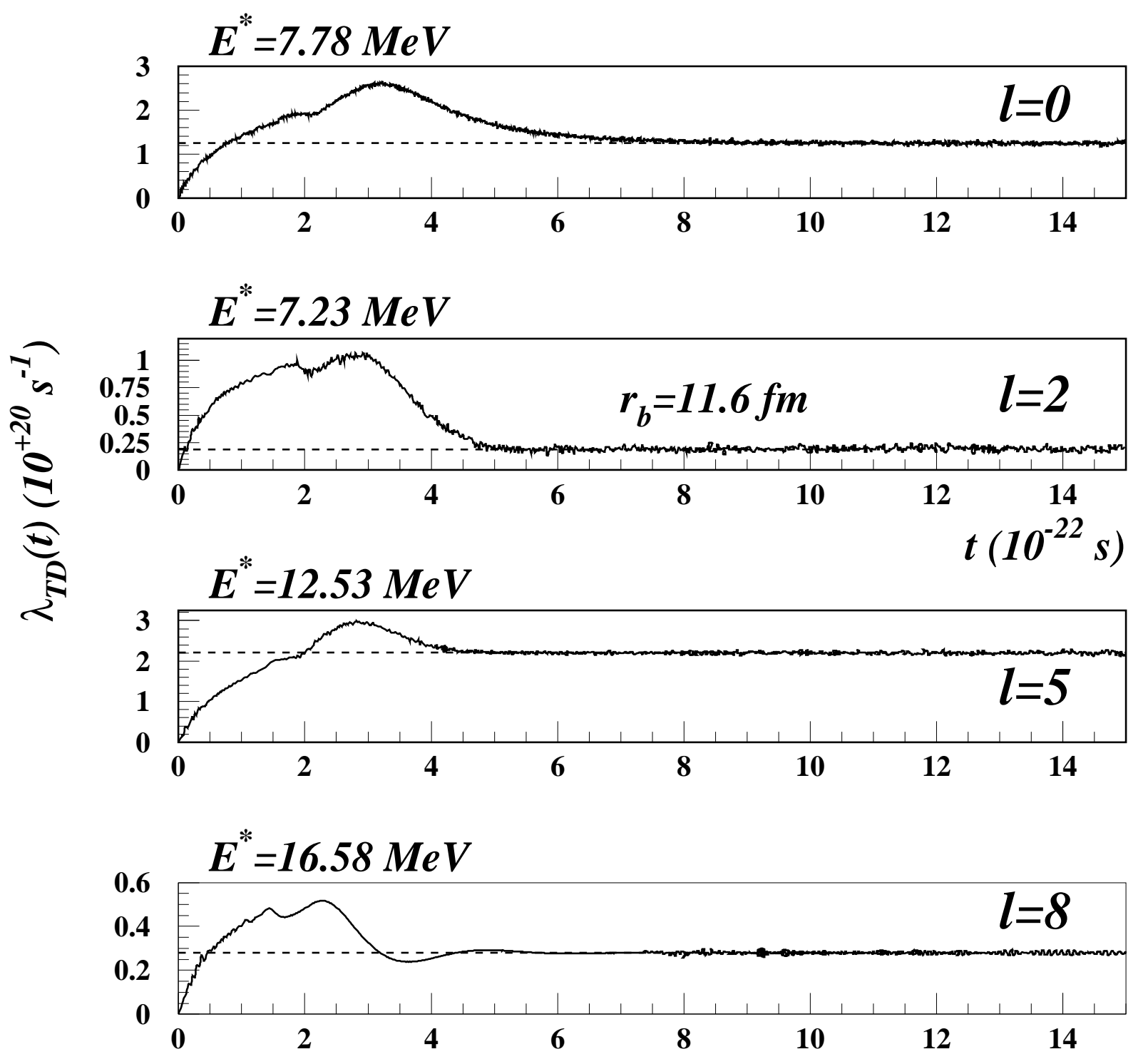

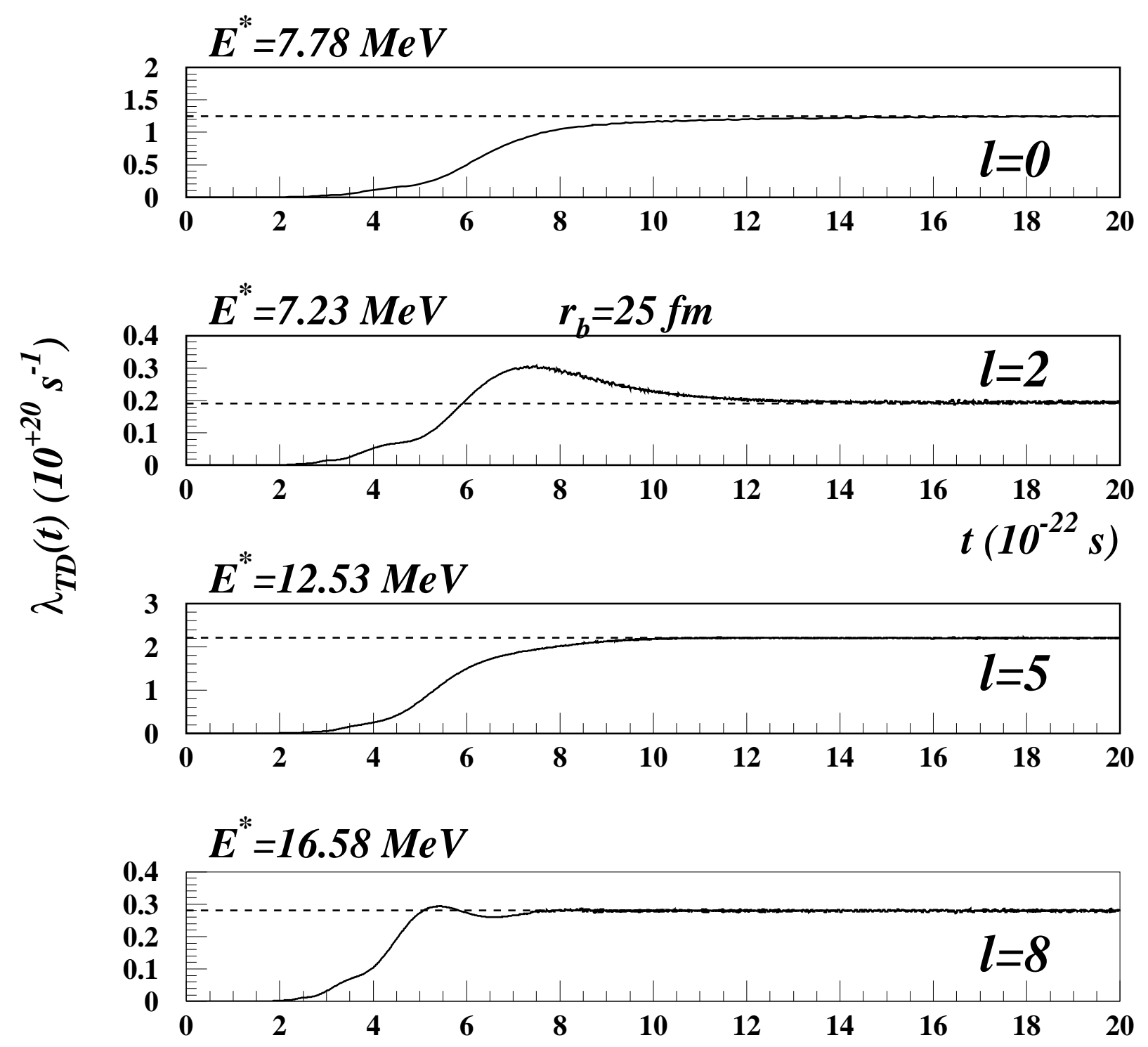

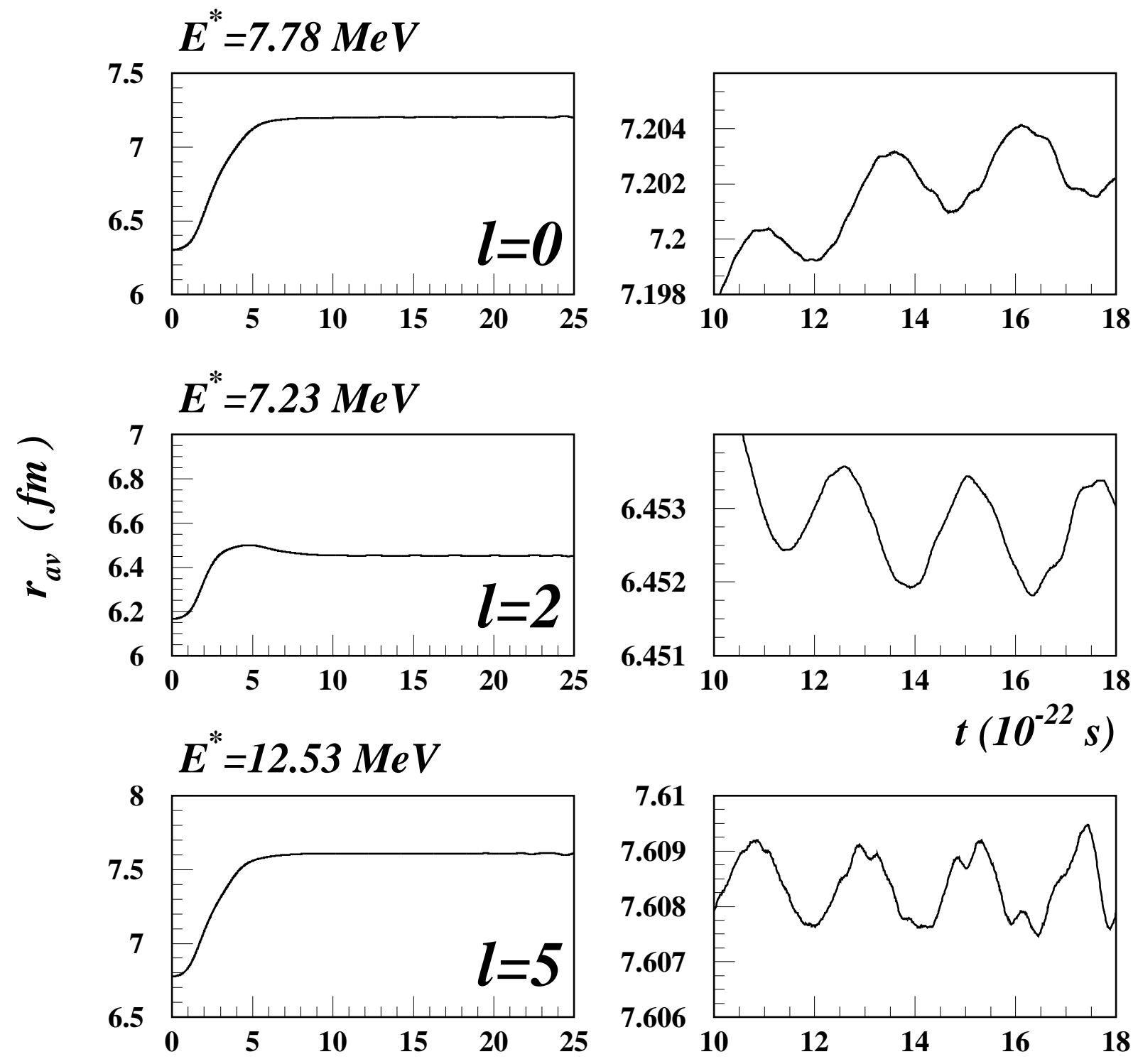

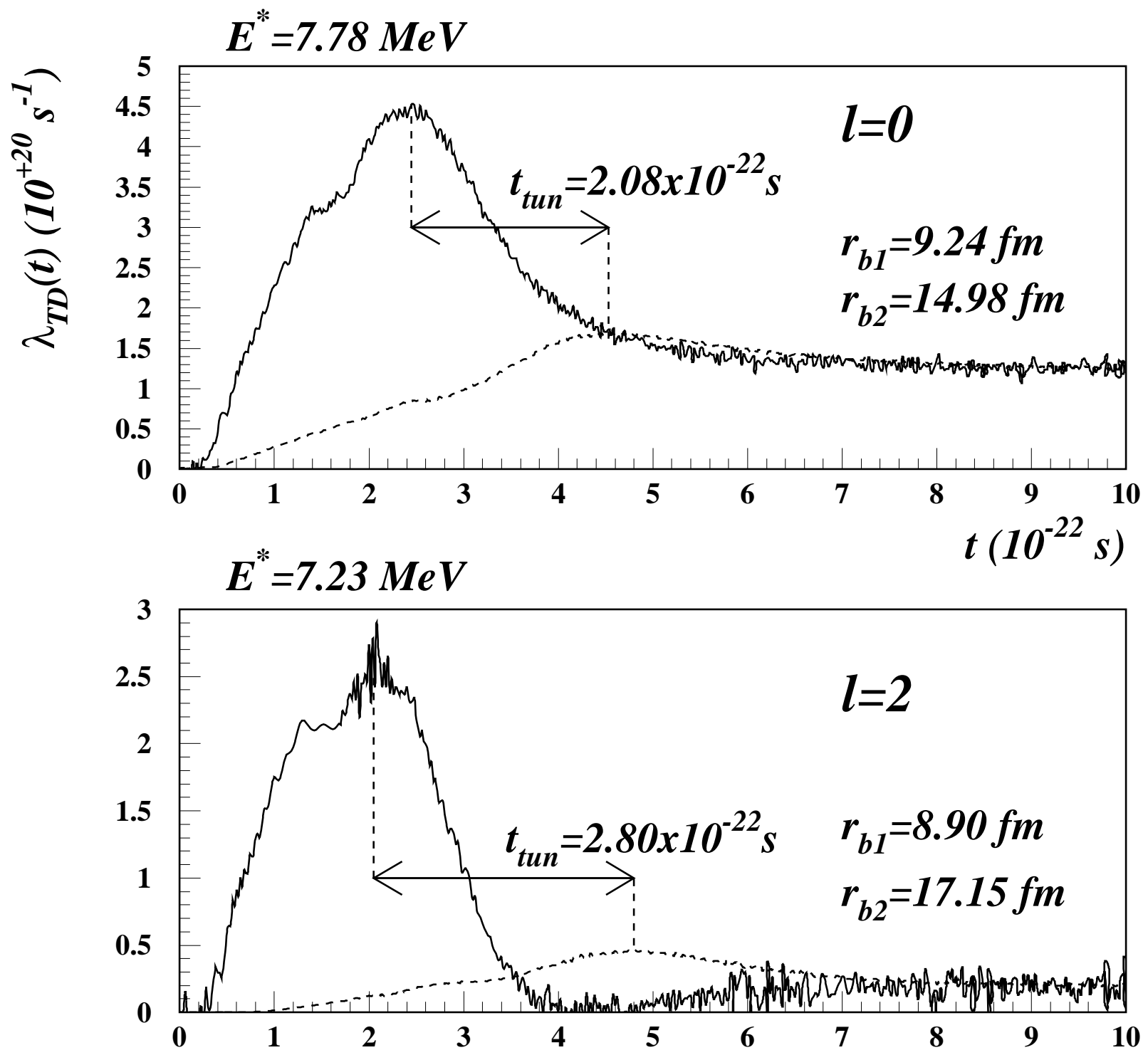

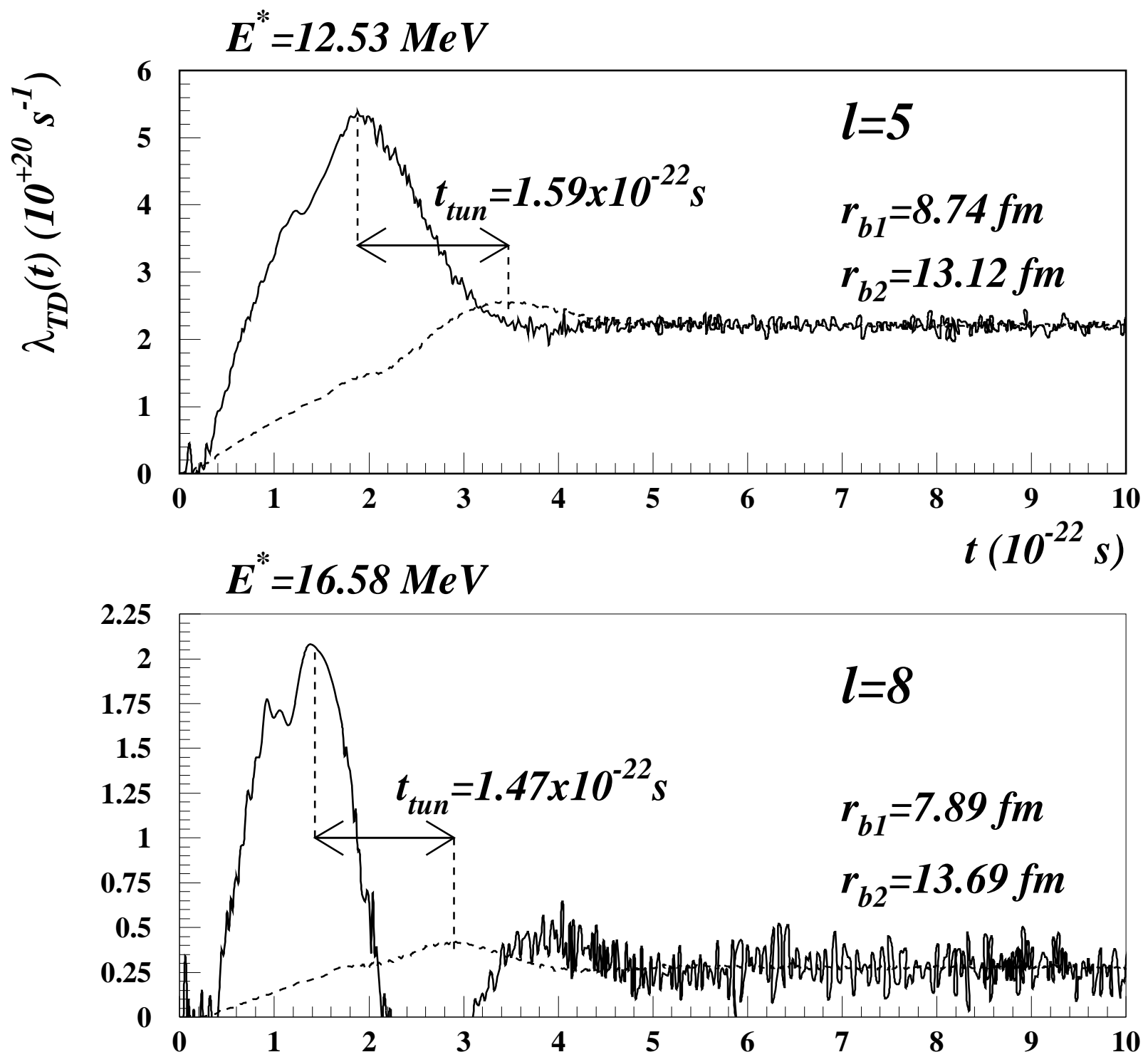